\title{
Joint Congestion Control and Channel Assignment Algorithm for Wireless Mesh Networks
}

\author{
Ganesh D \\ Assistant Professor \\ Department of Information Technology \\ Sree Vidyanikethan Engineering College \\ Tirupati-517 102, India
}

\author{
Venkata Rama Prasad V \\ Professor and Head \\ Department of Information Technology \\ Sree Vidyanikethan Engineering College \\ Tirupati-517 102, India
}

\begin{abstract}
Congestion control in wireless networks is an important issue to be addressed. Solutions exist for single radio, single channel assignments. However, congestion control in wireless radio, multichannel wireless mesh networks play a significant role in the network communications. This problem is more complex because multiple radio channel assignment and traffic monitoring per channel are both to be jointly solved. In this work, we present a solution to solve both the problems by dividing the problem into two sub problems. One part solves the congestion control and the other part solves the channel assignment sub problem. We solve the congestion control sub problem by distribution of traffic to a set of radio paths. This solution provides channel congestion information which is used to solve channel assignment sub problem. This is an iterative procedure which increases the overall network utilization. Compared to the previous works in multi radio wireless mesh networks, we show that our approach yields significant improvement in the network utilization.
\end{abstract}

Keywords: congestion control, traffic distribution, radio paths

\section{INTRODUCTION}

Mesh networks can achieve greater speed by spreading out multiple radios in orthogonal channels. These networks also have the potential for interference mitigation (via) use of multiple channels, which introduces flexibility to allocate resources spatially and controls congestion. In this work, we address the joint congestion control and channel assignment problem using an iterative, decomposition approach. This approach first determines a congestion-control driven channel assignment, and then for a given assignment, achieves the best distribution of traffic over possible combinations of radios. The channel assignment also ensures a high utility. We derive the feasibility conditions for congestion control problem in a given channel, which provides significant awareness of the actual congestion limits. Then we solve channel assignment sub problem which takes information from congestion control sub problem. We derive that a new channel assignment yields an increase in the network utilization.

First, for the congestion control sub problem, we account for multi radio, multi channel nature of the network. We reduce the traffic by distributing them to appropriately selected set of radio paths. For different radio paths this also provides a solution for traffic distribution problem. When convergence takes place this solution provides the transmission rate for each radio-to-radio link, which operates in different channels, under the interference and congestion conditions. Second, we use the above solution of congestion control to solve the channel assignment sub problem. Lagrange' Multipliers are used to interact with the two sub problems which can (1) locate the deficiencies of the previous iteration and motivate new changes (2) provide a local classification of the channels. These also impact the channel assignment on the global network fairness objective. The results in turn determine the new interference conditions and shape the congestion control problem of the next iteration. This approach can achieve significant network utilization and a wide range of fairness objectives.

\section{RELATED WORKS}

In the Context of wired networks we will study congestion control as a utility Maximization problem [8][11]. Studies of wireless networks using the utility maximization frame work include congestion control under asymetrics due to carrier sense[6]; joint design of congestion control and power control[4], incorporation of clique-feasibility constrains [23], joint design of congestion control and scheduling [5][9].The joint problem addressed is unique in that it is discrete and combinatorial in nature. In wired networks, we use utility maximization models for multipath routing [7] [10], but in wireless networks it does not incorporate spatial resource allocation aspect. The channel allocation in multiradio networks has been studied with an objective of load-aware, interferenceavoiding channel assignments [12] [13].We consider fairness objectives and channel assignments together with throughput maximization and routing. Recent approaches employ exhaustive search for the channel assignment problem. We propose a joint solution for both congestion control and channel assignment algorithm interacting with a multi-radio, multichannel congestion controller. Section II describes the network model and the problem statement. Section III proposes the feasibility conditions for the proposed model. Section IV describes the channel assignment and the eligibility conditions for the model. Section $\mathrm{V}$ discusses the Algorithm implementation. Section VI provides the Experimental Results and section VII gives conclusions of this work.

\section{NETWORK MODEL}

We consider a static wireless mesh network with a set of nodes denoted by $N$, where $N=|N|$. Each node $n \in N$ is equipped with $\mathrm{M}_{\mathrm{n}}$ identical radios. These nodes are connected with links $\varepsilon$, with $\mathrm{E}=|\varepsilon|$. The graph $(\mathrm{N}, \mathrm{E})$ is referred to as the network graph. Each link consists of radio logical links L, formed 
between end points. The set of these logical links are denoted $\mathrm{L}$ with $\mathrm{L}=|\mathrm{L}|$. The data transmission through these links must be with a constant rate $c_{\mathrm{L}}$. This Network consists of $\mathrm{K}$ orthogonal channels of equal bandwidth. An instance of a channel assignment to the radios of the network is denoted by $\pi=\left\{K_{n, i}, i=1, \ldots \ldots . M_{n}, n=1, \ldots . N\right\}$.where the radio $i$ of node $n$ is operating at channel $k_{n, i}$. Finally, the incoming traffic to a radio of a node can be immediately forwarded for the transmission to a different radio of the same node. We consider a set of sources $\mathrm{s}$ with $\mathrm{S}=|\mathrm{s}|$ in the given network. The utility of the source at average rate $X_{s}$ is expressed by a well known family of utility functions:

$u\left(x_{s}\right)=\left\{\begin{array}{l}w_{s} \frac{x_{s}^{1-\alpha}}{1-\alpha}, \text { if } \alpha \neq 1 \\ w_{s} \log x_{s}, \text { otherwise }\end{array}\right.$

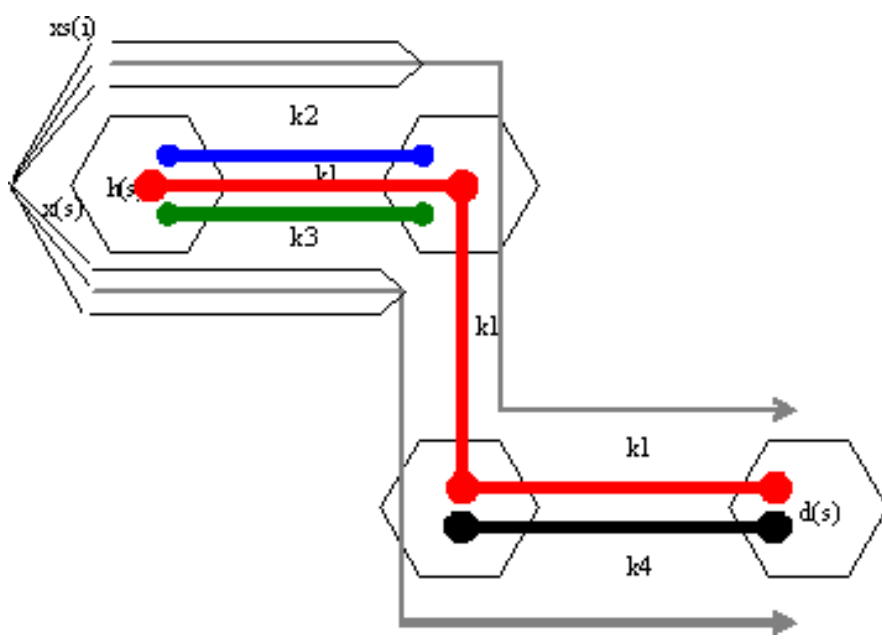

Figure 1: Construction of set of paths

Where $\mathrm{u}($.$) is strictly concave, non-decreasing, twice$ differentiable function. The fairness region depends on the priority parameters $w=\left\{w_{s}, s=1, \ldots . . s\right\}$ and parameter $\alpha$. Here $\alpha=0$, leads to throughput maximization, $\alpha=1$ to proportional fairness, $\alpha=2$ harmonic mean fairness and $\alpha=\infty$ represents min-max fairness. Each source $\mathrm{S}$ is associated with an origin-destination node pair denoted by $\left(h_{s}, d_{s}\right)$. We consider a fixed node-to-node routing, expressed by binary variables $R_{l, s}$ that are equal to 'one' if route of source s is using link $l$ and 'zero' otherwise. We denote D the maximum route size and denoted $\pi$, the space of all acceptable channel assignments.

\section{A. Problem Statement}

Let $\mathrm{P}$ denote the traffic distribution options in the network for a given channel assignment and $\sigma_{j}$ the available capacity of resource $\mathrm{j}$. The joint congestion control and channel assignment problem can be described as follows:

\section{B. $M R M C-C C-C A$}

$$
x_{s}(p)_{\pi \in \prod}^{\max } \sum_{s=1}^{S} U\left(x_{s}(p(\pi))\right)
$$

such that, $\sigma_{j}(\pi, x, P(\pi)) \geq 0, \forall_{j}$

The difficulty in solving channel assignment problem is that it forms integer variables and determines channel participation of radios and links. We mainly use a decomposition approach which divides the problem into two sub problems. The congestion control sub problem uses a fixed channel assignment and accounts for conditions that help in distributing traffic up to the congestion limits of network resources. Based on the computed source rates, the channel assignment sub problem utilizes Lagrange's multipliers and link utilization from congestion control sub problem. The two sub problems are solved until termination.

\section{CONGESTION CONTROL}

Here we limit the congestion control sub problem to fixed channel assignment $\pi$. The main goal of this problem is to derive rate adaptations and exploit transmissions from multi radios and different channels. This is achieved by constructing a set of radio paths for traffic distribution and by deriving an appropriate set of feasibility conditions for traffic to share channels and time slots.

\section{A. Path Construction}

In multiradio networks we have multiple channel assignments to spatially distribute the network capacity. Here the total incoming traffic at each intermediate is arriving from multiple radios and should be split to each of the outgoing links. We construct a set of radio-to radio paths for each node-to-node route $S$ as follows: Starting with link e adjacent to source $S$, we create one path $p$ for each common channel between its end points $\operatorname{tr}(\mathrm{e})$, rcv(e).For subsequent links of route e',for each common channel between its end nodes, we append the corresponding radio-toradio link to all the paths constructed for the previous link. This results in set of paths $p_{s_{2}}$ for each source $S$. our congestion design distributes the traffic across a set of the paths $p_{\mathrm{s} \text {. for each }}$ source $S$. The_number of paths $p_{s=\mid} p_{s}$ for each route $S$ is equal to product of common channels at each link along the route. The number is upper bounded by $\left(\max _{i \in n} M_{i}\right)^{D}$ where D is the maximum route length in the network. Every node is within D hops of a gateway and in typical Deployments D is rarely greater than five to six hops.

A source $\mathrm{S}$ perceives Utility $\mathrm{U}(\mathrm{Xs})$ when data is transmitted from $\mathrm{h}(\mathrm{s})$ to $\mathrm{d}(\mathrm{s})$ at total rate Xs. Rate Xs is the aggregate traffic achieved by transmission to each radio path $p \in p_{s}$ with rate $x_{s, p} \quad$ hence $\quad x_{s}=\sum_{p=1}^{p_{s}} x_{s, p}$ we denote $x_{s}=\left\{x_{s, p}, p=1, \ldots . . p_{s}\right\}$ the source distribution vector and by $\mathrm{X}=\left\{x_{s}, s_{d}, \ldots s\right\}$ the network distribution vector. In addition, we use the binary routing variables $R_{l}, s, p$ indicating if radio-to-radio link $l$ is used by path $\mathrm{P}$ of source $\mathrm{S}$ (or) not. 


\section{B. Feasibility Conditions}

We use the generic contention graph $\mathrm{CG}_{0}$ to describe interference relationships in the network graph. Each vertex in $\mathrm{CG}_{0}$ corresponds to node-to-node link in the network graph and each edge corresponds to two interfering links in the network graph.let $N_{c l}^{0}$ be the maximum cliques in $\mathrm{CG}_{0}$, because all channels use this $\mathrm{CG}_{0}$. We replicate this for $\mathrm{K}$ times, resulting in a total of $N_{c l}=K * N_{c l}^{0}$ maximum cliques. The network distribution factor $\mathrm{X}$ should impose normalized aggregate load on each clique $\Phi_{j}$ not to exceed a normalized capacity $C_{j}$ :

$$
\sum_{s=1}^{p} \sum_{p=1}^{p_{s}} \sum_{l=1}^{L} R_{l, s, p} F_{l, j} \frac{x_{s, p}}{c_{l}} \leq c_{j}, \forall j=1, \ldots . . N_{c l}
$$

where binary variables $F_{l, j}$ depend on the radio channel assignments and indicate radio link $l=1, \ldots, \mathrm{L}$ belong to clique $\mathrm{j}$, $\mathrm{j}=1, . ., N_{c l}$. The clique capacities $\mathrm{Cj}$ should guarantee the existence of a time slot schedule realizing the radio link loads included by X. Our clique formulation implies that cliques in each channel will be scheduled independently in the time domain. The clique based formulation introduces the complexity of computing all maximum cliques in a graph which is generally an NP-Complete problem and a time consuming computation in practice. Given radio paths and feasibility conditions, we formulate the congestion control sub problem as the following utility maximization ratio.

\section{C. $M R M C-C C$}

$$
\max \sum_{s=1}^{s} u\left(\sum_{p=1}^{p_{s}} x_{s, p}\right)
$$

Such that,

$$
\sum_{s=1}^{p} \sum_{p=1}^{p_{s}} \sum_{l=1}^{L} R_{l, s, p} F_{l, j} \frac{x_{s, p}}{c_{l}} \leq c_{j}, \forall j=1, \ldots . . N_{c l}
$$

Here each source $S$ is physically located at a given node from multiple combinations of traffic distribution in its path set $\mathrm{P}_{\mathrm{s}}$. Our radio path based formulation enables convergence to a unique solution for multipath routing problems in wireless networks [10][14][8]. The objective function is modified with small penalty quadratic terms,

$$
-\delta \sum_{s=1}^{S} \sum_{i=1}^{p_{s}}\left(x_{s, p}\right)^{2}
$$

Where $\mathrm{S}$ is a small positive constant. Exact solutions to MRMC$\mathrm{CC}$ can be achieved with the use of proximal optimization theory [3]. More precisely, an additional variable $Z_{s, p}$ is associated with each $x_{s, p}$, and the transformed problem is as follows:

\section{D. $M R M C-C C$ exact}

$$
\max \sum_{s=1}^{S} U\left(\sum_{p=1}^{p_{s}} X_{s, p}\right)-\delta \sum_{s=1}^{S} \sum_{p=1}^{p_{s}}\left(X_{s, p}-Z_{s, p}\right)^{2}
$$

The solution to MRMC-CC exact can be found by considering LaGrange's multipliers $\lambda$ associated with each constraint. The procedure can be summarized as follows [10]:

At each iteration $\mathrm{K}$,

(i) The distribution of traffic of source S,to the radios of the path $\mathrm{P}$ is given by:

$X_{s, p}(K+1)=\arg \max \left\{U\left(\sum_{p=1}^{p_{s}} X_{s, p}\right)-\sum_{j=1}^{N_{d}}\left\{\lambda_{j}^{*} * R_{l, s, p} F_{l, j} \frac{X_{s, p}}{c_{l}}\right\}-\delta \sum_{i=1}^{p_{s}}\left(X_{s, p}-Z_{s, p}\right)^{2}\right\}$

$X_{s}(K+1)=\sum_{p=1}^{p_{s}} X_{s, p}$

(ii) For $\mathrm{j}=1, \ldots, N_{c l}$ the Lagrange's multipliers are updated

$\lambda_{j}(K+1)=\left[\lambda_{j}(k)+\gamma\left(\sum_{s=1}^{S} \sum_{p=1}^{P_{s}} \sum_{l=1}^{L} R_{l, s, i} F_{l, j} \frac{X_{s, p}}{c_{l}}-C_{j}\right)\right]^{+}$

Where $\gamma$ is a sufficiently small step size. The variables $Z_{s, p}$ converted to $\bar{X}_{s, p}$ of the corresponding variables $X_{s, p}$ and the process repeats until convergence.

\section{CHANNEL ASSIGNMENT}

In change of discrete constraint coefficient $F_{l j}$ which will produce new The above solution of congestion control yields optimal paths and source rates for a given channel assignment. For very small networks, exhaustive search is not feasible due to large number of channel assignments. Hence, we are proposing this solution which solves congestion control and channel assignment sequentially. Given a solution $X$ of the congestion control sub problem that results from channel assignment $\pi$, we seek new channel assignment $\pi^{\mid}$that will yield a congestion control sub problem $X^{\mid}$of higher aggregate network utility in the next iteration. The new channel assignment $\pi^{\downarrow}$ may remove traffic from highly congested resource or add bandwidth to the resource. The above approach would not necessarily yield a channel assignment that will result in the increase of network utility function. The key idea of our channel assignment algorithm uses Lagrange's multipliers to identify most congested Cliques. Within these Cliques local Channel Modifications are done resulting in new links may be added or deleted. From the optimization view point, if we perform Channel modifications it results constraint for the next iteration. Here we have to find out that which channel modifications will guarantee to increase network utility function. Our algorithm will find out such channels by utilizing the traffic vector $\mathrm{X}$ of congestion control sub problem. We introduce a channel assignment algorithm that incorporates the channel modifications and conditions. The channel modifications may be link based or radio based. Link based modifications involve switching both radios of a radio link to a different channel. Radio based modifications involve switching only a single radio to a different channel. Link based modifications result in a more link switching channels. 


\section{A. Link Deletion}

A radio of link $l$ in Clique $\mathrm{j}$ switches from channel $K_{l}$ to $K^{\mid}$.The new channel $K^{\mid}$should be different than channels assigned to other radio links of node-to-node $\operatorname{link} e_{l}$ where radio link $l$ belongs. This modification results in deletion of the radio link $l$ and all adjacent links of its switched radio channel $K_{l}$.At the same time this modification may result in addition of new link adjacent to the radio.

\section{B. Link Reinforcement:}

Let $e_{l}^{l}$ be the node-to-node link where a radio link $l^{\mid}$of clique $\mathrm{J}$ belongs. A radio of a parallel radio link $l$ that also belongs to $e_{l}$ switches from its channel $k_{l}$ to channel $k^{\mid}$.This modification results in deletion of links adjacent to radio of channel $k_{l}$ and addition of links adjacent to this radio in channel $\boldsymbol{k}^{\mid}$.

\section{Channel Assignment Algorithm:}

The proposed algorithm selects channel reassignments at each iteration of the congestion control channel assignment loop. Its input is the link loads $x_{l}$, Lagrange's multipliers of the congestion control sub problem and current channel assignment $\pi$.Its output is a new channel assignment $\pi^{\mid}$that aims at higher aggregate network utility in the next iteration. This algorithm visits all Cliques $\mathrm{j}$ in descending order of their Lagrange's multipliers $\lambda_{j}$.For each clique $j$, the algorithm searches overall local channel modifications interms of link deletions and link reinforcement and identifies $I_{j}$ set of eligible modifications. If no modifications are found the algorithm proceeds to next Clique, otherwise the algorithm terminates by selecting the modifications that yields the maximum movement of aggregate traffic for both link deletion and link reinforcement. The output of the above step will serve as input for the congestion control sub problem of the next iteration. If no eligible channel modification is found for any clique, the algorithm terminates at NULL output. This also terminates congestion control/channel assignment loop since no further improvements can be guaranteed by channel assignment

\section{ALGORITHM IMPLEMENTATION}

In this section we evaluate the performance of our algorithm and compare it in [1], which introduces an optimization frame work to jointly determine routes and channel assignments that optimize aggregate throughput. We first discuss the algorithm implementation and then evaluate the performance.

\section{A .Implementation}

We have implemented our joint congestion control and channel assignment framework using custom simulator in Java 2.0. And run on a personal computer with Pentium IV cpu at $2.6 \mathrm{GHZ}$. The following algorithms are used

\section{B. Maximum Clique Enumeration Algorithm}

We will use this algorithm at network initialization to find out maximum Cliques given node locations and interference relationships. This results in an NP-complete problem for arbitrary graphs but there exists efficient algorithm for graphs in wireless networks. We can also use this in large topologies implemented by a greedy heuristic technique.

\section{Congestion Control Algorithm}

This algorithm provides a fixed channel assignment and a set of computed radio paths. This algorithm is iterative and requires simple algebraic operations of lower complexity.

\section{Channel Assignment Algorithm}

This algorithm provides low complexity since it only involves local searches in most congested cliques. The eligibility condition allows us to select good channel modifications thru algebraic manipulators without verifying congestion control sub problem.

\section{E. Performance Evaluation}

The approach followed in [1] can be referred as ABL05. Consider a given set of source destination pairs, both approaches source rates $x_{s}$. ABL05 also computes node-to-node paths between source destination pairs. This approach is based on linear programming formulation and yields a feasible source rate of $\lambda^{*}$ subjected to fairness constraint as $x_{s}=\lambda^{*} * w_{s}$ where $w_{s}$ is the weighted demand vector. Our approach also uses the utility based formulation which has a wide range of fairness objectives. Multiple node-to-node paths increase the complexity. We pre compute the shortest path algorithm according to Dijkstra's shortest path algorithm. We compare the two approaches using equal weights $w_{s}$ for all sources, as we cannot determine the optimal weights in advance. To illustrate this we use a grid topology scenario that provides uniform interference patterns and Chaska topology that provides nonuniform interference.

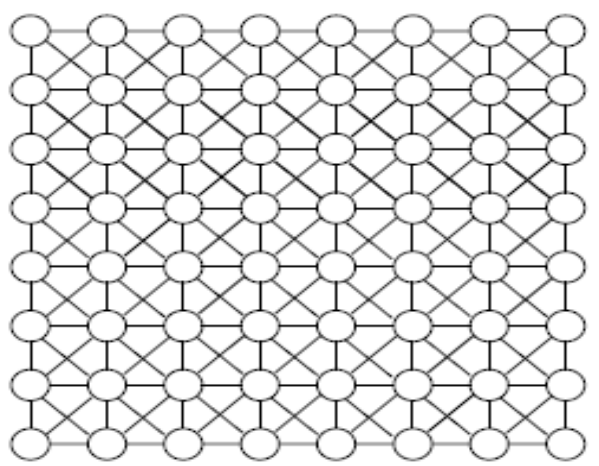

Figure 2(a). Grid Topology

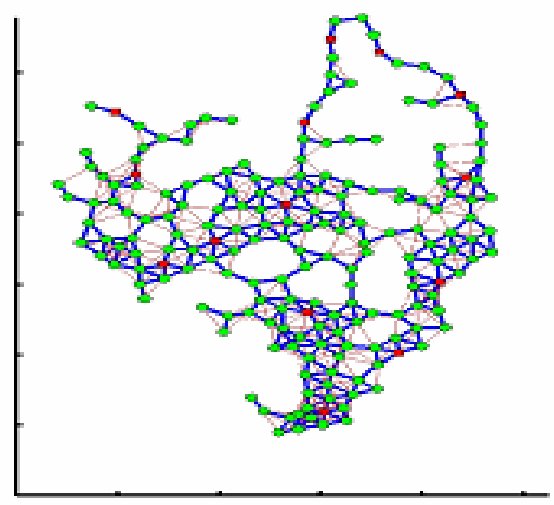


Figure 2(b). Chaska Topology

\section{F. Grid scenario}

This scenario is shown in figure 2(a) and uses custom simulator, nodes have equal number of 802.11 a radios $\mathrm{M}$, with rates range from $6 \mathrm{Mbps}$ to $54 \mathrm{Mbps}$ with maximum transmission rates ranges from $30 \mathrm{~m}$ to $90 \mathrm{~m}$ respectively. Maximum interference range is $180 \mathrm{~m}$; The distance between two adjacent points in $8 \mathrm{X} 8$ grid is $58.5 \mathrm{~m}$ and 60 nodes are placed in the grid randomly.here number of radios per node is $\mathrm{M}$ and number of channels are K.for each (M.K) number of gateways are 2 to12.The reported average and minimum source rates are averages across all gateways configured.

\section{G. Chaska scenario}

Figure 2(b) depicts the 194 node topology mesh network deployed in Chaska. The transmission range is $250 \mathrm{~m}$ and interference range is $400 \mathrm{~m}$. The bit rate of this topology we considered is $11 \mathrm{Mbps}$. This is a large topology with non-uniform density. so that we use 24 out of 194 nodes as gateways. The reported average and minimum source rates are averaged across all the traffic matrices.

\section{EXPERIMENTAL RESULTS}

We simulated the Grid and Chaska topologies for various (M, $\mathrm{K})$ values. The results obtained are shown in figures 3(a) and 3(b). We observe that ABL05 severely underutilizes the mesh network in both the scenarios. The ABL05 rate is lower than average MRMC-CC rate due to two reasons. First, the fairness constraint produces equal source rates in non-regular topologies when $w_{s}=1$. Second, the Clique based constraint is more conservative than link based constraint used in ABL05. In grid scenario, the under utilization of ABL05 is more due to link based constraint, because regularity of grid topology favors the choice when $w_{s}=1$. In Chaska topology this becomes more severe. On the other hand, MRMC-CC yields higher average rate than ABL05 and the difference increases for more resources. For the grid scenario, we run

the MRMC-CC for the network wide rate sum optimization ( $\alpha=0$ ) which yields higher average rate. Hence, the average rate of MRMC-CC for $\alpha=1$ is comparable to average rate at $\alpha=0$. Hence in this grid scenario network resources are utilized in an efficient manner.

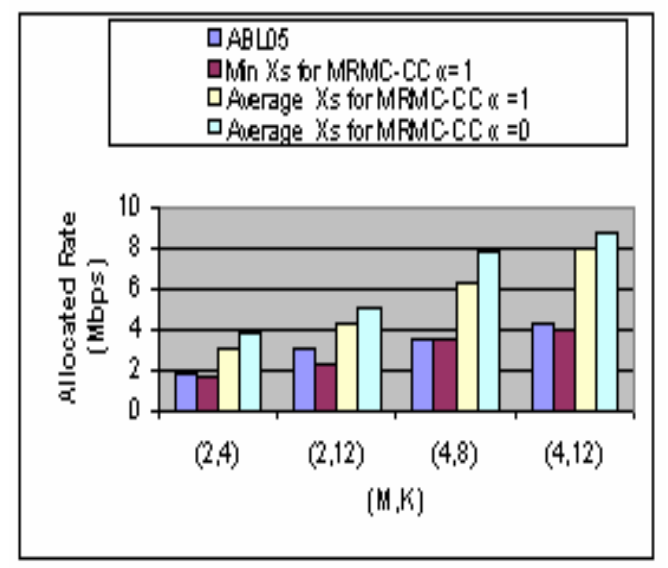

Figure.3 (a). Grid Performance Evaluation

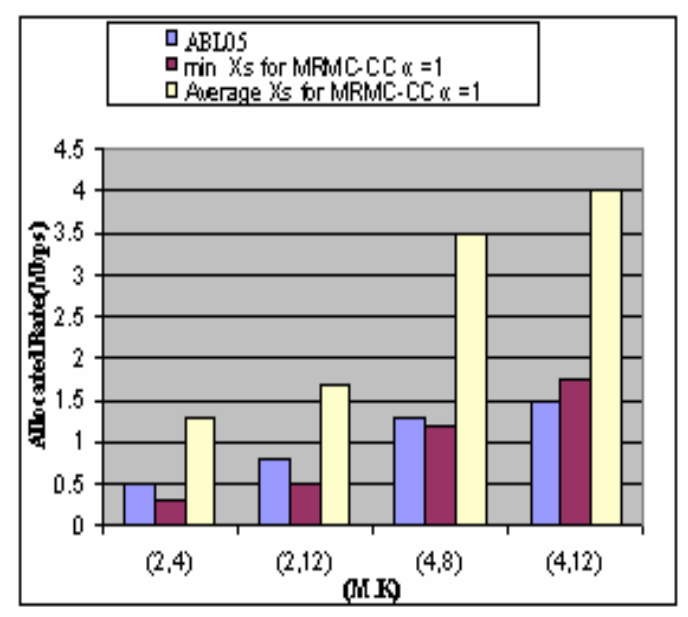

Figure.3 (b). Chaska Performance Evaluation

\section{CONCLUSIONS}

In this work we have jointly addressed the problems of congestion control and channel assignment in multi radio, wireless networks with a single algorithm. The key contribution of this paper is due to traffic distribution to a set of paths that characterizes the radio and channel transmission capabilities. We follow a decomposition approach where congestion control and channel assignment problems are solved iteratively. We used this iterative approach on Grid and Chaska topologies and obtained good results. This approach outperforms existing approaches and yields rates and channel assignments that achieve high network utilization

\section{REFERENCES}

[1] M. Alicherry, R. Bhatia, and Li., "Joint channel assignment and routing for throughput optimization in multi-radio wireless mesh networks," Proc. ACM mobicom, cologne, Germany, Sept. 2005.

[2] http://www.ilog.com/products/cplex

[3] D. P. Bertsekas, Nonlinear programming, Athena scientific, 2 ed., 2003.

[4] M. Chiang, "Balancing transport and physical layers in wireless multihop networks: joint design of congestion control and power control," IEEE Journal on selected areas in communication, 23(1):104-116, Jan 2005.

[5] A. Eryilmaz and R. Srikant, "Fair resource allocation in wireless networks using queue length based scheduling and congestion control," Proc. IEEE INFOCOM, Miami. FL, USA, March 2005.

[6] V. Gambirozaand E. Knightly, "Congestion control in CSMA-based networks with inconsistent channel states," Proc. IEEE/ACM international wireless internet conference, Boston, August 2006

[7] H.Han, R. Srikant, "Overlay TCP for multipath routing and congestion control," ENS-INRIA ARC TCP Workshop, Paris, Nov. 2007.

[8] F. Kelly, A. Maullo, and D. Tan, "Rate control in communication networks: proportionality fairness and stability," Jl. of the operational research society, 49:237252, 1998. 
[9] X. Lin and N. B. Shroff, "Joint rate controlling and scheduling in multihop wireless networks," Proc. of Control and Decision conference, Dec. 2006

[10] X. Lin and N. B. Shroff, "Utility Maximization for communication networks with multipath routing," IEEE Trans. on Networking, 51(5):766-781, May 2007.

[11] S. Low and D. Lapsley, "Optimization flow control,Basic algorithm and convergence," IEEE Trans. On Networking,7(6):861-874,Oct. 2004.

[12] K. RamaChandran and M. Buddhikot, "Interference aware channel assignment in multi radio wireless mesh networks," Proc. IEEE INFOCOM, Spain, April 2006.

[13] A. Raniwala and T. Chiueh, "Centralised algorithms for multichannel wireless mesh networks," ACM SIGMOBILE Mobile computing and communications Review, April 2006.

[14] X. Gao, T. Nandagopal, and T. Kim, "Achieving MAC layer fairness in wireless packet networks," Proc. ACM MobiCom, Boston, August 2000 .

\section{AUTHORS BIOGRAPHY}

Mr. D. Ganesh received his B.Tech degree in Information Technology from JNT University, Hyderabad in 2006 and M.Tech degree in Computer Science and Engineering from Acharya Nagarjuna University in 2010.During the period 200607 he worked as Assistant Professor in Information Technology department at AITS, Rajampet, India. Since 2007, he is working as Assistant Professor in IT Department at Sree Vidyanikethan Engineering College, Tirupati, India. He has Published 6 papers in national and International conferences. His current research interests are computer networks, wireless networks, Object oriented design and unified modeling. He is a member of ISTE, CSI.
Dr. Rama Prasad V Vaddella received the M.Sc(Tech.) degree in Electronic Instrumentation from Sri Venkateswara University, Tirupati in 1986 and M.E degree in Information Systems from BITS, Pilani, India in 1991. During the period 1989-1992 he worked as Assistant Lecturer in Computer Science at BITS, Pilani. From 1992 to 1995, he worked as Lecturer in Computer Science and Engineering and as Associate Professor from 1995 to 1998 at RVR \& JC College of Engineering, Guntur, India. Since 1998, he is working as Professor and Head of Information Technology department at Sree Vidyanikethan Engineering College, Tirupati, India. He was awarded the Ph.D degree in Computer Science by J.N.T. Univeristy, Hyderabad, during 2007 for his thesis in Fractal Image Compression. He is also holding the additional position of Chairman, Board of Studies in Computer Science, and Vice- Principal at Sree Vidyanikethan Engineering College (Autonomous Institution under JNT University, Anantapur, India). He has also worked as a short time Research Assistant at Indian Institute of Science, Bangalore during the year 1986 . He has published about 15 papers in national and international conferences and 06 papers in International journals, edited books, and refereed conferences. $\mathrm{He}$ is also a member of the editorial review board for 05 International journals in Computer Science and Information Technology. He is also a member of the review committee for 05 international journals. His current research interests include computer graphics, image processing, computer networks, computer architecture and neural networks. He is a member of IEEE, ISTE, IACSIT, IAENG and CSI. 